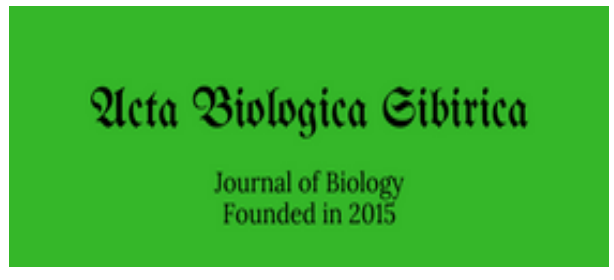

Altai State University

wWw.asu.ru

ISSN 2412-1908

Acta Biologica Sibirica, 2019, 5(1), 30-32

\title{
New data on the distribution of fungus gnats (Diptera: Bolitophilidae, Keroplatidae) in Tyva Republic (Russia, Siberia)
}

\author{
Sh.V. Dongak, E.Yu. Subbotina, Yu.V. Maximova
}

Tomsk State University, Lenin Ave. 36, Tomsk634050 Russia,E-mail: Allodia@sibmail.com

\begin{abstract}
An annotated list is given and the distribution of 13 species of fungus gnats of the families Bolitophilidae and Keroplatidae, collected from Tuva in Eastern Siberia, is provided. As a result of our study, 11 species were added to the list of fungus gnats of Tuva
\end{abstract}

Key words: Diptera; Bolitophilidae; Keroplatidae; fungus gnats; Eastern Siberia; Tyva Republic; Tuva

\section{Introduction}

Bolitophilidae and Keroplatidae (Diptera) are two families within the large superfamily Sciaroidea, whose representatives (excluding Sciaridae and Cecidomyiidae) are united under the common name fungus gnats. A characteristic feature of fungus gnats is the trophic connection of their larvae with fungi; so, the fungus gnats are mainly associated with the forest zone in their distribution. The Bolitophilidae and Keroplatidae families in the adjacent territories were studied in Altai Republic (Zaitzev, 1994), Kuznetsky Alatau (Ostroverkhova \& Maksimova, 2000; Maximova, 2002) and in the southern taiga subzone in the south of Tomsk Region (Subbotina, 2014). Fragmentary information about Tuva fauna is only found in the work of A.l. Zaitzev (1994). Due to the lack of information on the fauna of fungus gnats of Eastern Siberia, the relevance of our work emerges.

\section{Material and methods}

The material was collected in the Tyva Republic (Todjinsky district, Sut-Khol district) from 19.06 to 30.08.2017 in five localities (Points No. 1-5). Dongak was the collector unless otherwise specified. Only males were used for determination. The material is stored at the Department of Zoology of Invertebrates of Tomsk State University.

The abbreviations used in the text to designate places of assembly are as follows:

Point N1. Tyva Republic, Todjinsky district, near the village of Toora-Khem (52²8'25.69" N / 96º6'54.98" E), h=950 m. Larch-birch forest: Betula pendula, Larix sibirica, Populus alba, Picea obovata, Rosa majalis, Rubus idaeus, Phleum pratensis, Dactylis glomerata, Carum carvi, Achillea millefolium, Rubus saxatilis, Fragaria vesca, Geranium sylvaticum, Poa trivialis, Vicia cracca, Carex acuta, Linaria vulgaris. Material was collected by using entomological net and yellow pan-traps.

Point N2. Tyva Republic, Todjinsky district, near the lake Tore-Khol (52³3'00.60" N / 96²12'07.28" E), h=1127 m. Spruce-larch forest: Picea obovata, Larix sibirica, Pinus sibiricaAbies sibirica, Rosa majalis, Equisetum sylvaticum, Carex acuta, Rubus saxatilis, Frigaria vesca, Plantago major, Campanula sibirica. Material was collected by using Malaise traps (19.07-01.08.2017).

Point N3. Tyva Republic, Todjinsky district, near the village of Toora-Khem (52³0'52.33" N / 96º6'24.71" E), h=925 m. Pine-spruce forest: Picea obovata, Pinus sylvestris, Pinus sibirica, Larix sibirica, Betula pendula, Carex acuta, Poa trivialis, Taraxacum officinale, Phleum pratensis, Alchemilla vulgaris, Plantago major, Euphrasia officinalis, Aegopodium podagraria, Centaurea scabiosa. Material was collected by using entomological net.

Point N4. Tyva Republic, Todjinsky district, near the lake Azas (52²3'10.90" N / 96²3'51.42" E), h=960 m. Birch-larch forest: Larix sibirica, Betula pendula, Picea obovata, Betula pendula, Salix acutifolia, Rosa majalis, Rosa acicularis, Ribes 
rubrum, Lonicera altaica, Stellaria bungeana, Vaccinium vitis-idaea, Trollius asiaticus, Cirsium helenoides, Crepis sibirica, Lilium pilosiusculum. Material was collected by using entomological net.

Point N5. Tyva Republic, Sut-Khol district, near the lake Sut Hol (51²9'23.23" N / 9109'47.09" E), h=2065 m. Larch forest: Larix sibirica. Rhododendron dahuricum, Lonicera altaica, Caragana arborescens и Caragana jubata. Rosa majalis, Vaccinium uliginosum, Pedicularis oederi, Oxytropis saposhnikovii, Pulsatilla campanella, Gagea serotina, Rhodiola rosea. Material was collected by using entomological net.

Systematics of Bolitophilidae and Keroplatidae, as well as information about their distribution are given in accordance with the online catalog Fauna Europaea (Chandler, 2005). Species identification was carried out, using keys to genera and species of A.I. Zaitzev (1994) and G.P. Ostroverkhova (1979).

The abbreviations used in the text in the distribution section are as follows:

Russian European part (EP): North-West (NW), Centre (C).

Western Siberia (WS): Tomsk Prov. (TK), Kuznetsky Alatau (KU), Altai (AL) (including Altai Rep. and Altai Terr.).

Eastern Siberia (ES): Khakassia Rep. (KS), Tuva Rep. (TU), Krasnoyarsk Terr. (KR), Irkutsk Prov. (IR).

Far East (FE): Khabarovsk Terr. (including Jewish Autonomous Region) (KH), Primorskii Terr. (PR), Sakhalin (SA), Kuril Islands (KU).

Other countries: JA - Japanese; NA - North America.

\section{Results}

\section{BOLITOPHILIDAE Winnertz, 1863 \\ Bolitophila Meigen, 1818 \\ Cliopisa Enderlein, 1936}

1. Bolitophila bispinosa Mayer, 1951

Material examined: 1 male (Point N1: 07.08.2017), 1 male (Point N5: 31.07.2017).

Distribution: Europe; Russia: EP, ES (KR, TU).

\section{Bolitophila glabrata Loew, 1869}

Material examined: 1 male (Point N1: 15.08.2017); 1 male (Point N4: 25.08.2017).

Distribution: Europe; Russia: NW, EP, WS (TK), ES (TU); FE (KH).

3. Bolitophila hybrida (Meigen, 1804)

Macrocera hybrida Meigen, 1804. Bolitophila fusca Meigen, 1818. Bolitophila nigrolineata Landr., Krivosheina, Zaitzev, Yakovlev, 1986, Bolitophila nigrolineata Landr., Stakelberg, 1969. Bolitophila nigrolineata Landr., Ostroverkhova, 1979 (Zaitzev, 1994).

Material examined: 1 male (Point N1: 30.08.2017)

Distribution: Europe; Russia: EP, WS (TK, KU, AL), ES (TU); FE (PR, KU); NA.

4. Bolitophila incisa Ostroverkhova \& Grishina, 1974

Material examined: 1 male (Point N1: 07.08.2017); 1 male (Point N1: 30.08.2017).

Distribution: Russia: WS (TK), ES (TU).

\section{Bolitophila nigrolineata Landrock, 1912}

Material examined: 1 male (Point N4: 25.08.2017)

Distribution: Europe; Russia: EP, WS (AL), ES (TU); FE (PR, SA).

6. Bolitophila occlusa Edwards, 1913

Material examined: 1 male (Point N1: 30.08.2017)

Distribution: Europe; Russia: EP, WS (KU, KS, AL), ES (TU); FE (PR, SA); JA.

7. Bolitophila pseudohybrida Landrock, 1912

Bolitophila triangulata Edwards, 1941.

Material examined: 2 males (Point N1: 19.06.2017)

Distribution: Europe; Russia: EP, WS (KU), ES (IR, TU); FE (SA, KU).

\section{KEROPLATIDAE Rondani, 1856}

Keroplatinae Rondani, 1856

Pyratula Edwards, 1929

8. Pyratula perpusilla (Edwards, 1913)

Material examined: 3 males (Point N1: 15.08.2017); 5 males (Point N1: 30.08.2017); 1 male (Point N2: 19.07 01.08.2017); 1 male (Point N3: 09.08.2017), 5 males (Point N4: 25.08.2017).

Distribution: Europe; Russia: ES (TU, IR).

\section{Orfelia Costa, 1857}

\section{Orfelia discoloria (Meigen, 1818)}

Platyura discoloria Meigen, 1818. Platyura diluta Loew, 1869. Platura discolor Zetterstedt, 1851.

Material examined: 1 male (Point N1: 15.08.2017)

Distribution: Europe; Russia: EP, WS (TK), ES (KR, TU); FE (PR). 


\section{Orfelia krivosheina A. Zaitzev}

Lit. data: Zaitzev, 1994: 95.

Distribution: Russia: ES (TU).

\section{Macrocerinae Rondani, 1856}

Macrocera Meigen, 1803

11. Macrocera phalerata Meigen, 1818

Macrocera maculipennis Macquart, 1826.

Lit. data: Zaitzev, 1994:72.

Distribution: Europe; ES (TU).

\section{Macrocera pilosa Landrock, 1917}

Lit. data: Zaitzev, 1994:73.

Distribution: Russia: EP, ES (TU).

\section{Macrocera stigmoides Edwards, 1925}

Material examined: 1 male (Point N5: 31.07.2017).

Distribution: Europe; Russia: NW, C, WS (AL), ES (TU).

\section{References}

Chandler, P.J. (2005). Fauna Europaea: Mycetophilidae. Fauna Europaea: Diptera, Nematocera. Retrieved June 2018 (as version 2.2) from http://www.faunaeur.org

Maximova, Yu.V. (2002). An additional list of the fungus gnats (Diptera, Sciaroidea, excluding Sciaridae) from Kuznetsk Alatau Mountains. An International Journal of Dipterological Research,13(3, 191-199.

Ostroverkhova, G.P. (1979). Fungus gnats (Diptera, Mycetophiloidea) of Siberia. Tomsk: Tomsk State University. (in Russian)

Ostroverkhova, G.P., \& Maksimova, Yu.V. (2000). A preliminary list of the fungus gnats (Diptera, Sciaroidea, excluding Sciaridae) from Kuznetsk Alatau Mountains. An International Journal of Dipterological Research. 11(3),145-155.

Subbotina, E.Yu. (2014). Fauna of fungus gnats (Diptera: Bolitophilidae, Keroplatidae, Mycetophilidae) of subtaiga of Western Siberia. Euroasian Entomological Journal, 13(1), 47-58. (in Russian)

Zaitzev, A.I. (1994). Fungus gnats of the fauna of Russia and adjacent regions. Part 1. Russian Academy of Sciences. Moscow: Nauka. (in Russian)

\section{Citation:}

Dongak, Sh.V., Subbotina, E.Yu., \& Maximova, Yu.V. (2019). New data on the distribution of fungus gnats (Diptera: Bolitophilidae, Keroplatidae) in Tyva Republic (Russia, Siberia). Acta Biologica Sibirica, 5 (1), 30-32.

Submitted: 20.11.2018. Accepted: 15.01.2019

cross ref http://dx.doi.org/10.14258/abs.v5.i1.5187

(C) 2019 by the authors. Submitted for possible open access publication under the terms and conditions of the Creative Commons Attribution (CC BY) license (http://creativecommons.org/licenses/by/4.0/). 